\title{
Preparation and Sensitivity Measurements of Graphene Oxide-RDX Composite
}

\author{
Mahmoud Y. Zorainy ${ }^{1}$, Waleed F. Aly ${ }^{1}$, Ahmed Elbeih $^{1, a}$, and Mohamed Gobara ${ }^{1}$ \\ ${ }^{1}$ Military Technical College, Kobry Elkobbah, Cairo, Egypt
}

\begin{abstract}
The effect of graphene oxide (GO) on the safety characteristics of 1,3,5-trinitro-1,3,5-triazinane (RDX) was studied in this work. Graphene oxide was prepared and was investigated to form a composite based on GO-RDX by solvent-antisolvent slurry technique. For comparison, different polymer bonded explosives (PBXs) based on RDX bonded by viton A, fluorel or polymethyl-methacrylate binders were studied and designed as RDX-Viton, RDX-Fluorel and RDX-PMMA respectively. Sensitivities to impact and friction of the presented samples as well as the pure RDX was measured. The ignition temperatures were determined and the activation energies were calculated by using the ignition delay method. Results of $\mathrm{x}$-ray diffraction and scanning electron microscope proved that RDX crystals were coated by a thin layer of GO. The impact sensitivity of GO-RDX composite is lower than that of the other studied samples while the friction sensitivity is slightly higher. The ignition temperature of GO-RDX was lower than the other studied samples which indicates that the GO caused accumulation of the decomposition gaseous products and accelerated the decomposition process of RDX. GO is an interesting candidate material to be used for coating the explosive crystals instead of the polymeric matrices.
\end{abstract}

\section{Introduction}

In the field of energetic materials, it is well known that the advanced explosives have high performance and in the same time high sensitivities [1-3]. The researchers are seeking to obtain modern explosives with high performance and high safety during handling and transportations [4-6]. The sensitivities of the explosives could be enhanced by controlling the crystal shapes and sizes [7,8]. Traditionally, the preparation of polymer bonded explosives (PBXs) was the optimum solution to increase the safety of the explosives without affecting their performance $[9,10]$. Many publications reported the preparation methods and the characteristics of the prepared PBXs $[11,12]$. The percentage of the polymer used to coat the explosive crystals has significant effect of the performance of the prepared PBXs [13]. The thermal behavior and decomposition kinetics of PBXs are important to produce and long life PBXs $[14,15]$. 1,3,5-trinitro-1,3,5-triazinane (RDX) is an interesting explosive which has several applications if the field of energetic materials [2,3]. The sensitivity of RDX is consider high and cannot be pressed as a pure materials. Graphene oxide (GO) is a two-dimensional (2D) material and can be obtained by oxidation of graphite powders [16]. GO has been widely applied in material sciences due to its large theoretical specific surface area and abundant oxygen-containing groups, which can significantly alter the properties of materials it is combined with $[17,18]$.

In this work, a method for preparation of GO-RDX composite is presented. The effect of coating the explosive crystals by 2 wt $\%$ of GO on the impact and friction sensitivities was discussed, in addition to its effect of the activation energy and the ignition temperature of the pure RDX. For comparison, selected PBXs have been studied.

\section{Preparation of graphene oxide (GO)}

GO was prepared by improved Hummers method with some modification. Preparation of GO was prepared by improved Hummers method, four steps has been followed to apply this method (30). 1.st step putting $90 \mathrm{~mL} \mathrm{H}_{2} \mathrm{SO}_{4}$ and $60 \mathrm{~mL} \mathrm{H}_{3} \mathrm{PO}_{4}$ in beaker $250 \mathrm{ml}$, adding $3.0 \mathrm{~g}$ Graphite in this beaker with stirring in ice bath for $30 \mathrm{~min}$, till the temp. Reach $0{ }^{\circ} \mathrm{C}$, adding slowly 20 $\mathrm{g} \mathrm{KMnO}_{4}$, and stirring for $1 \mathrm{~h}$. The second step was removing the beaker from ice bath and putting it hotplate at $60{ }^{\circ} \mathrm{C}$ for $24 \mathrm{hr}$. then adding a $500 \mathrm{ml}$ of cold deionized water to the forms avoid temperature increase $>50{ }^{\circ} \mathrm{C}$, adding $30 \mathrm{ml} \mathrm{H}_{2} \mathrm{O}_{2}$ the color turn from brown to yellow, adding $500 \mathrm{ml} \mathrm{DW}$ for $24 \mathrm{hr}$. the last step based on removal of the water superannuated adding $500 \mathrm{ml} \mathrm{DW}$ put the baker in ultrasonic bath for $1 \mathrm{hr}$ at $40 \mathrm{GHz}$ and wash by HCL 5\% and DW using centrifuge at $10000 \mathrm{rpm}$ until pH reach 6 and dry the precipitation by vacuum oven at $60{ }^{\circ} \mathrm{C}$ for $12 \mathrm{hr}$.

\section{Preparation of GO-RDX composites}

\footnotetext{
${ }^{\mathrm{a}}$ Corresponding author : elbeih.czech@gmail.com
} 
The formation of GO-RDX composites was prepared In brief; in a beaker of $250 \mathrm{ml} 10 \mathrm{mg}$ of the prepared GO was dispersed in 50 $\mathrm{ml} \mathrm{DMF}$ and sonicated for $60 \mathrm{~min}$. Then, $0.5 \mathrm{~g}$ of RDX was dissolved in $50 \mathrm{~mL}$ DMF with magnetic stirring and added to the GO suspension and assisted ultrasonic vibration, allowed for stirring for $2 \mathrm{~h}$ at $40^{\circ} \mathrm{C}$. After RDX and GO was completely dissolved in DMF solvent, the mixture was dropped into the anti-solvent coldwater under magnetic stirring. The product was separated by centrifugation at $5000 \mathrm{rpm}$ and washing by methanol and drying in vacuum oven at $50^{\circ} \mathrm{C}$ for $12 \mathrm{hr}$. The GO-RDX composite was prepared in with a theoretical mass fraction of GO of $2 \%$. RDX was recrystalline in a similar process without addition of GO.

\section{$4 \mathrm{X}$-ray diffraction}

The XRD patterns of GO, RDX, and GO-RDX by using a fully computerized X-ray diffractometer, Shimadzu XRD-6000 with Cu radiation $\lambda=1.54056 \mathrm{~A}^{\circ}$ was carried out at National Center for Radiation Research and Technology, Egyptian Atomic Energy Authority. The X-ray tube was operated at $40 \mathrm{kV}$ and $30 \mathrm{~mA}$ anode current throughout the measurements. The above operation conditions were maintained during all the relevant measurements. The $2 \theta$ scan range from $4^{\circ}$ to $90^{\circ}$ continuous scanning with scan speed $8(\mathrm{deg} / \mathrm{min})$ and preset time $0.15 \mathrm{sec}$ was used for the entire $2 \theta$-range.

Many parameters can be concluded from X-ray analysis such as crystal structure, atomic arrangement, phase orientation, lattice parameters and grain size. The samples of pure GO, RDX and as-prepared GO-RDX composites were examined by XRD. As shown in Fig. (1), a sharp peak at 2 theta $=10.20$ is related to GO powder with d-spacing $=8.0427 \AA$. Moreover, d-spacing value was increased due to the insertion of function groups between graphite layers. This indicated the high oxidation applied to graphite during graphene oxide preparation. These results highly agree with the previous literature of preparation GO [18]. The major diffraction pattern of RDX is almost the same as that of GO-RDX composites (such as $12.8^{\circ}, 17.14^{\circ}$ and $28.9^{\circ}$ ) indicated that interaction between GO and RDX.

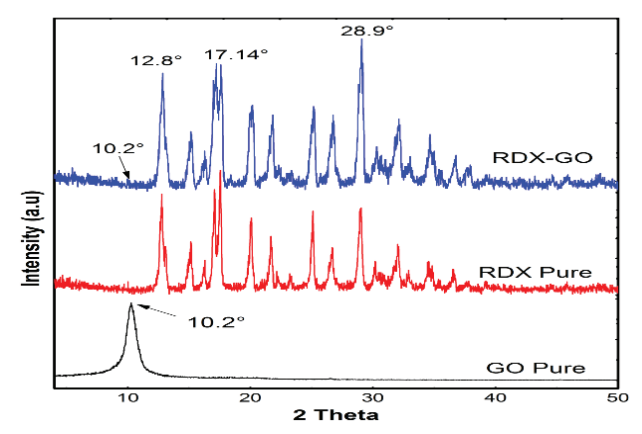

Figure 1. XRD of the studied materials.

\section{Crystal morphology study}

Scanning electron microscope (SEM) model Inspect S obtained from FEI company was used to study the crystal morphology of RDX and GO-RDX crystals. Figure 1 presents the SEM photos of the pure RDX crystals with magnification.

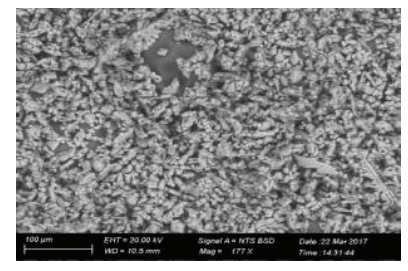

(a)

Figure 2. SEM photos of RDX crystals.

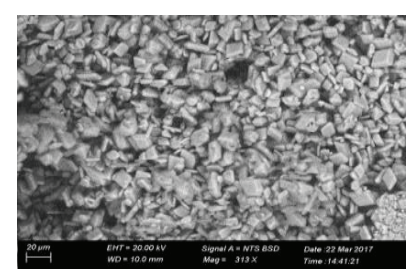

(a)

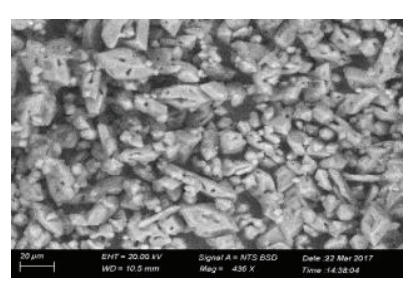

(b)

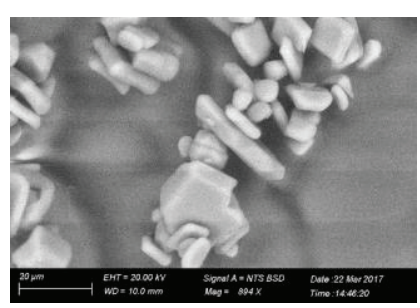

(b)

Figure 3. SEM photos of the GO-RDX crystals. 
As shown in Fig. 2, pure RDX crystals are in the microscale and the crystals have particle size less than $100 \mu \mathrm{m}$. The RDX crystals have non-uniform shapes and there are cracks on the surface of the crystals. Also the surface of the crystals are rough and that is the reason of the high sensitivity of the pure RDX. The morphology of the prepared GO-RDX is shown in fig. 3, where the crystal have nearly the same size as the pure RDX. It means that the coating of RDX by GO does not affect the size of the used RDX. The crystals of the prepared GO-RDX have a smooth surface where the cracks of the pure RDX disappeared due to covering of the crystals by GO and it confirms that GO are precipitated on the surface of RDX crystals. Also the GO caused decrease to the sharp edges of the RDX crystals, as a result it could decrease the sensitivity of the pure RDX due to the modification of the morphology of the crystals. It is also clear that addition of $2 \mathrm{wt} \%$ of GO affect the morphology of RDX crystals.

\section{Impact sensitivity measurements}

The impact sensitivity of the prepared GO-RDX in addition to the studied PBXs based on RDX was determined by a standard impact tester with exchangeable anvil (Julius Peters [19]) where a $2 \mathrm{~kg}$ drop hammer was use. The tested samples had a volume of $50 \mathrm{~mm}^{3}$. The results of the Probit analysis technique [20] was used to determine the probability of initiation of the tested samples. The sensitivity to impact was reported as the impact energy required for $50 \%$ probability of initiation of the samples and is reported in Table 1.

\section{Friction sensitivity measurements}

The friction sensitivity was obtained by using BAM friction test apparatus with applying the standard test conditions [19]. 0.01g of the studied explosive was spreaded on the surface of the porcelain plate and different loads were used to change the normal force between the porcelain pistil and the plate. Detection of the sample initiation was observed through sound, smoke appearance, or through smell of the decomposition products. The Probit analysis was used to determine the probability of initiation of the samples. However, only $50 \%$ of initiations was reported as the normal friction force required for initiation and reported in Table 1 as the friction sensitivity.

Table 1. The sensitivity measurements of the studied explosives.

\begin{tabular}{|c|c|c|c|c|}
\hline No. & Explosive type & $\begin{array}{c}\text { Impact } \\
\text { sensitivity } \\
(\mathbf{J})\end{array}$ & $\begin{array}{c}\text { Friction } \\
\text { sensitivity } \\
(\mathbf{N})\end{array}$ & $\begin{array}{c}\text { Ignition } \\
\text { temperature } \\
\left({ }^{\mathbf{}} \mathbf{C}\right)\end{array}$ \\
\hline 1 & RDX [21] & 5.6 & 120 & 215 \\
\hline 2 & GO-RDX & 13.4 & 268 & 213 \\
\hline 3 & RDX-viton [10] & 10.6 & 326 & 216 \\
\hline 4 & RDX-fluorel [10] & 10.8 & 352 & 217 \\
\hline 5 & RDX-PMMA [10] & 11.9 & 240 & 212 \\
\hline
\end{tabular}

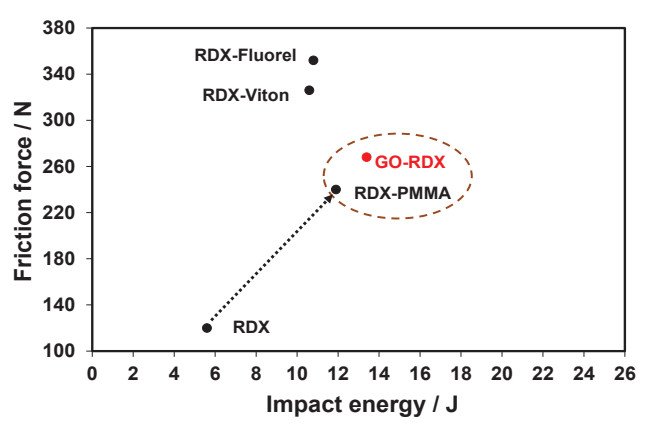

Figure 4. Comparison between the impact and friction sensitivities of all the studied samples.

The results of the impact and friction sensitivities are presented on fig. 4. It is clear that RDX has a high impact sensitivity while GO-RDX has the lowest impact sensitivity of all the studied samples (13.4 J) even that GO-RDX contains $98 \mathrm{wt} \%$ of RDX while the other PBXs have $9 \mathrm{wt} \%$ of RDX. It means that GO has a great influence on decreasing the impact sensitivity of RDX. This result might be due to the effect of the GO sheets which can dissipate the impact energy and decrease the effect of the potential energy on the RDX crystals and prevent the hot spot formation. On the other side, the friction sensitivity of GO-RDX is higher than RDX-Viton and RDX-Fluorel. This might be due to the characteristics of the fluorinated binders which has great effect on decreasing the sliding friction on the explosive itself. While GO-RDX has lower friction sensitivity than RDX-PMMA. It means that the results of GO-RDX were the optimum compared with the studied PBXs. 


\section{Ignition temperature and ignition delay measurements}

Chilworth deflagration test apparatus was used to determine the ignition temperature and ignition delay of the samples. Three test tubes contained $0.1 \mathrm{~g}$ from each sample were placed vertically into heating device at the same moment where the temperature was increased by fixed heating rate of $5{ }^{\circ} \mathrm{C} \mathrm{min}^{-1}$. Once the sample was ignited, the ignition temperature was recorded where the mean value was recorded in table 1. Ignition delay was obtained by increasing the temperature of the heating block over the ignition temperature and determine the delay time required for ignition of the samples at different temperatures. The time required for ignition of each sample is presented in table 2 at different isothermal temperatures. The kinetic parameters can be obtained by using the measurement of the ignition delay method as reported in literature [22]. Arrhenius equation was used to determine the activation energy of the studied samples by plotting a linear relationship between ln (time to ignition, $t_{\text {ig, }}$ ) and $1000 /$ temperature, $\mathrm{T} / \mathrm{K}$, of each sample as shown in Fig. 5. The activation energies of the samples were obtained from the slope of the curve and recorded in table 2 .

Table 2. Ignition delay time $\left(\mathrm{t}_{\mathrm{id}}\right)$ and activation energy $\left(\mathrm{E}_{\mathrm{a}}\right)$ of the studied samples.

\begin{tabular}{|c|c|c|c|c|c|c|c|c|c|c|}
\hline \multirow{2}{*}{ Sample } & \multicolumn{7}{|c|}{$\mathbf{i}_{\text {id }} / \mathbf{s}$ at temperatures / ${ }^{\mathbf{C}}$} & \multirow{2}{*}{$\mathbf{E} / \mathbf{k J ~ m o l}^{\mathbf{1}}$} & \multirow{2}{*}{$\mathbf{R}^{\mathbf{2}}$} \\
\hline GO-RDX & 250 & 260 & 270 & 280 & 290 & 300 & 310 & 320 & 131.3 & 0.9918 \\
& 33 & 21 & 10 & 6 & 4 & 2 & - & - & & \\
\hline RDX- & 250 & 260 & 270 & 280 & 290 & 300 & 310 & 320 & 74.8 & 0.9549 \\
Fluorel & 39 & 25 & 16 & 10 & 8 & 7 & 6 & 5 & & \\
\hline \multirow{2}{*}{ RDX-Viton } & 250 & 260 & 270 & 280 & 290 & 300 & 310 & 320 & 81.5 & 0.9832 \\
& 41 & 26 & 17 & 11 & 9 & 7 & 6 & 4 & & \\
\hline RDX- & 250 & 260 & 270 & 280 & 290 & 300 & 310 & 320 & 96.2 & 0.9649 \\
PMMA & 34 & 23 & 13 & 7 & 6 & 5 & 4 & 2 & & \\
\hline
\end{tabular}

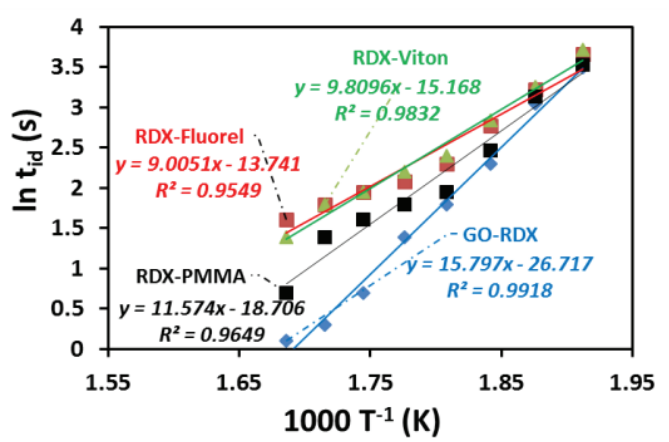

Figure 5. A linear relationship between $\ln$ (tig) and $1000 \mathrm{~T}-1$ for all the studied samples.

From fig. 5, it is clear that GO-RDX has the highest activation energy of all the studied samples while RDX-fluorel and RDXviton have very close results. It means that the increase of the activation energy of GO-RDX might be due to the interaction forces between the graphene oxide with the crystals of RDX where the decomposition is difficult. Also the fluorinated binders (viton and fluorel) have almost the same effect on the pure RDX.

\section{Conclusion}

From this study it was concluded that the method used for preparing composite of GO-RDX produced crystals with regular shape and smooth surface which enhanced the sensitivity characteristics of GO-RDX. A composite of RDX with 2 wt\% of GO has sensitivity to impact lower than PBXs containing $9 \mathrm{wt} \%$ binder. GO-RDX has friction sensitivity in the same level of RDXPMMA and higher than RDX-viton. These results clarified that GO has obvious effect on decreasing the sensitivity due to uniaxial compression while it has lower effect on shear slide with a fixed volume (sensitivity to friction). The activation energy of GORDX obtained by the ignition delay is higher than the other studied PBXs which means that the interaction between GO and RDX was strong and required a higher activation energy than the traditional PBXs. GO is a candidate to replace the binder systems which are used to decrease the sensitivity of explosives.

\section{References}

1. A. Elbeih, J. Pachman, S. Zeman, W.A. Trzcinski, M. Suceska, Propellants, Explosives, Pyrotechnics, 38, 238 (2013).

2. H.-H. Licht, Propellants Explosives Pyrotechnics, 25, 126 (2000).

3. S. Zeman, A. Elbeih, Z. Akštein, Hanneng Cailiao/Chinese Journal of Energetic Materials, 19, 8 (2011).

4. Q.-L. Yan, S. Zeman, P.E. Sánchez Jiménez, T.-L. Zhang, L.A. Pérez-Maqueda, A. Elbeih, Journal of Physical Chemistry C, 118, 22881 (2014).

5. A. Elbeih, S. Zeman, M. Jungova, Z. Akstein, Central European Journal of Energetic Materials, 9, 131 (2012).

6. T.M. Klapötke, Chemistry of high-energy materials, (Walter de Gruyter GmbH \& Co KG, 2015). 
7. A. Elbeih, A. Husarova, S. Zeman, Central European Journal of Energetic Materials 8, 178 (2011).

8. Q.-L. Yan, S. Zeman, A. Elbeih, A. Zbynek, Central European Journal of Energetic Materials, 10, 509 (2013).

9. A. Singh, M. Kumar, P. Soni, M. Singh, A. Srivastava, Defence Science Journal, 63, 622 (2013).

10. A. Elbeih, S. Zeman, M. Jungova, P. Vávra, Z. Akstein, Propellants, Explosives, Pyrotechnics, 37, 676 (2012).

11. Q.-L. Yan, S. Zeman, R. Svoboda, A. Elbeih, Thermochimica acta, 547, 150 (2012).

12. Q.-L. Yan, S. Zeman, F.-Q. Zhao, A. Elbeih, Thermochimica acta, 556, 6 (2013).

13. A. Elbeih, M. Mohamed, T. Wafy, Propellants, Explosives, Pyrotechnics, 41, 1044 (2016).

14. M. Abd-Elghany, T. Klapötke, A. Elbeih, S. Zeman, Journal of Analytical and Applied Pyrolysis, 126, 267 (2017).

15. A. Elbeih, S. Zeman, J. Pachman, Central European Journal of Energetic Materials, 10, 339 (2013).

16. C. Zhang, X. Cao, B. Xiang, J. Phys. Chem. C, 114, 22684 (2010).

17. Z. Li, Y. Wang, Y. Zhang, L. Liu, S. Zhang, RSC Advances, 5, 98925 (2015).

18. J. Wang, B. Ye, C. An, B. Wu, H. Li, Y. Wei, J. Energ. Mater, 34, 235 (2016).

19. M. Suceska, Testing Methods of Explosives (Springer, Heidelberg, 1995).

20. D. Finney, Probit Analysis (Cambridge University, third edition, 1971).

21. A. Elbeih, T. Wafy, T. Elshenawy, Central European Journal of Energetic Materials, 14, 77 (2017).

22. A. Elbeih, M. Abd-Elhgany, T. Elshenawy, Acta Astronautica, 132, 124 (2017). 